\title{
Políticas públicas de apoyo educativo y configuración de trayectorias escolares
}

Public Policies of Educational Support and Configuration of School Trajectories Politiques publiques d'appui éducatif et configuration des parcours scolaires Políticas públicas de apoio educativo e configuração de trajetórias escolar

Fecha de recepción: 5 DE NOVIEMBRE DE 2015/Fecha de aceptación: 25 DE JULIO DE 2017/Fecha de disponibilidad en línea: 1 DE DICIEMBRE DE 2017

Encuentre este artículo en http://magisinvestigacioneducacion.javeriana.edu.co/

\begin{abstract}
Resumen
Este artículo de investigación presenta un estudio sobre las políticas públicas españolas de apoyo educativo y su relación con la configuración de las trayectorias escolares, concretamente en la Comunidad de Madrid. Se ha empleado una metodología cualitativa a partir de un enfoque etnográfico. Los resultados muestran que los programas fomentados por las políticas de apoyo aquí contempladas, bajo determinadas condiciones, pueden suponer una oportunidad para estudiantes con una trayectoria escolar devaluada. Las conclusiones sugieren que estas políticas son valoradas, apropiadas e implementadas de forma diferenciada por los actores implicados, lo que da lugar a experiencias escolares y vitales que pueden suponer tanto una oportunidad de mejora en relación con las trayectorias de los estudiantes como una interpelación del mismo funcionamiento escolar.
\end{abstract}

\section{Palabras clave}

Política educacional; fracaso escolar; educación

compensatoria; justicia social; derecho a la educación

Para citar este artículo / To cite this article / Pour citer cet article / Para citar este artigo

Monarca, Héctor (2017). Políticas públicas de apoyo educativo y configuración de trayectorias escolares. magis, Revista Internacional de Investigación en Educación, 10 (20), 69-84. https://doi.org/10.11144/Javeriana.m10-20.ppae 
Keywords

Educational policy; school failure; compensatory education; social justice; education rights

\section{Mots clés}

Politique éducationnelle; échec scolaire; éducation compensatoire; justice sociale; droit à l'éducation

\section{Abstract}

This article presents the results of a research on the public policies of educational support and its relation with the configuration of the school trajectories. A qualitative methodology has been used based on an ethnographic approach. Results show that the programs promoted by the support policies contemplated here, under certain conditions, can be an opportunity for students with a devalued educational trajectory. Conclusions suggest that these policies are valued, appropriate and implemented in a differentiated way by the actors involved, giving rise to school experiences that can be both an opportunity for improvement in relation to the trajectories of students as an interpellation of the same school performance.

\section{Résumé}

Cet article de recherche présente les résultats d'une recherche par rapport aux politiques publiques d'appui éducatif et son rapport à la configuration des parcours scolaires. On q utilisé une méthodologie qualitative à partir d'une perspective ethnographique. Les résultats montrent que les programmes développés par les politiques d'appui ici considérées sous certaines conditions, peuvent supposer une opportunité pour les étudiants avec un parcours dévalué. Par ailleurs, les conclusions montrent que ces politiques sont prises en compte, appropriées et implémentées d'une façon différenciée par les acteurs impliques, en faisant place aux expériences scolaires et vitales qui peuvent donner tantôt une opportunité d'améliorer par rapport aux parcours des étudiants qu'une interpellation du même fonctionnement scolaire.

\section{Palavras-chave}

Política educacional; fracasso escolar; educação compensatória; justiça social; direito à educação

\section{Resumo}

Este artigo de pesquisa apresenta os resultados de uma investigação sobre as políticas públicas de apoio educativo e sua relação com a configuração das trajetórias escolares. Empregou-se uma metodologia qualitativa a partir de um enfoque etnográfico. Os resultados mostram que os programas fomentados pelas políticas de apoio aqui contempladas, em determinadas condições, podem supor uma oportunidade para estudantes com uma trajetória escolar subvalorizada. As conclusões indicam que estas políticas são valorizadas, apropriadas e implementadas de forma diferenciada pelos atores implicados, dando lugar a experiências escolares e vitais que podem supor tanto uma oportunidade de melhora em relação às trajetórias dos estudantes como uma interpelação do mesmo funcionamento escolar. 


\section{Introducción}

El actual sistema educativo tiene un origen claramente diferenciador y elitista (Fernández-Enguita, Mena-Martínez \& Riviere-Gómez, 2010; Monarca, 2011). Nuestros sistemas educativos — hijos de la modernidad ilustrada, de la revolución industrial, de la configuración de los estadosnación y de sus procesos de democratización — vienen siendo interpelados desde diversas ópticas, al mismo tiempo que se los somete a nuevas o renovadas demandas. La escuela está inevitablemente atravesada por las contradicciones de la sociedad en la que vivimos: los rasgos y exigencias de una sociedad democrática, el funcionamiento del mercado y la división social del trabajo, como fuerzas organizadoras de la sociedad, entre otras; pero solo estas tres se acompañan de una gran variedad de supuestos que plantean cada vez más desafíos y tensiones.

Por otra parte, como coinciden varios investigadores y pensadores de diversas disciplinas, las representaciones en torno a la sociedad están en un proceso de profundo cambio; con importantes consecuencias tanto para el pensamiento como para la acción. En esta línea, François Dubet y Danilo Martuccelli (2000, p. 165) consideran que estamos asistiendo al "estallido de la representación clásica del individuo y de la acción"; y enmarcan aquí lo que ellos denominan la desinstitucionalización, proceso por el cual —ligado a la descomposición de las representaciones en torno a lo social- las instituciones ya no son capaces de responder a las exigencias para las que fueron creadas. La sociedad, tal como venía siendo representada hasta hace unas pocas décadas, ya no existe, se ha deshilachado y, en este contexto, las instituciones ya no pueden cumplir de forma clara los propósitos para los que fueron creadas, las instituciones siguen atadas a los mandatos fundacionales mientras asumen otros nuevos productos de los cambios. Este escenario, sostienen los autores recién mencionados, hace que vivamos bajo un sentimiento de crisis continua, como añoranza de un pasado en el que las cosas sí funcionaban. Este mito del pasado mejor, sostiene Gabriela Diker (2010), supone una mirada apocalíptica, ya que no hay retorno a ese pasado; se refuerza así una visión permanente de "tragedia educativa", según la cual "todo está cada vez peor en las escuelas" (Diker, 2010, p. 151), y nos advierte de los nocivos efectos de este tipo de razonamiento que atentan contra los avances democratizadores de la educación, ya que este aludir constante a ese tiempo pasado nos lleva a una escuela elitista y selectiva.

No obstante las añoranzas y del mito del pasado mejor, un análisis comparativo en perspectiva histórica y que tome en cuenta el funcionamiento global del sistema educativo en relación con toda la población, nos permite apreciar que, muy lejos de las imágenes catastrofistas, muchas veces claramente intencionadas, hay importantes logros democratizadores y un claro beneficio de sectores anteriormente excluidos o que transitaban por circuitos segregados (Dubet, 2005), algo que también se puede comprobar al analizar la estadística disponible (Ministerio de Educación, Cultura y Deporte, 2015). El sistema ha cambiado, está en continuo cambio; en algunos aspectos ha mejorado significativamente: acceso a las distintas etapas educativas, más titulados en educación secundaria obligatoria (ESO), más estudiantes que acceden a la universidad; se han instalado y se siguen instalando concepciones democratizadoras. Pero al mismo tiempo persisten evidencias de aquel pasado añorado en términos de concepciones y de prácticas. Las tendencias democratizadoras avanzaron sobre la escuela fragmentada, elitista (Pericacho-Gómez, 2014); pero no la reemplazaron, edificaron sobre ella, se mezclaron con ella (Monarca,
Descripción del artículo | Article description | Description de l'article | Artigo descrição

Este artículo de investigación presenta los resultados del proyecto Estudio de las políticas públicas de acción afirmativa en educación. Se ha empleado una metodología cualitativa a partir de un enfoque etnográfico. Los resultados muestran que los programas fomentados por las políticas de apoyo aquí contempladas, bajo determinadas condiciones, pueden suponer una oportunidad para estudiantes con una trayectoria escolar devaluada. 
2011). Avanzar hacia un sistema educativo que sea plenamente inclusivo sigue siendo un desafío vigente en la actualidad.

Las políticas como intento de otorgar direccionalidad a lo real se mueven entre estas tensiones; de esta manera, sus intentos y sus prácticas están plagados de representaciones muchas veces contradictorias. Así, las políticas entendidas como "la dimensión de contingencia y apertura que posibilita la decisión individual y colectiva sobre el mundo social y la subversión, incipiente o radical, de sus principios estructurantes" (Vitar, 2006, p. 27), se mueven en un amplio campo de intereses y tensiones más o menos evidentes. Las políticas para la inclusión educativa se concretan en un marco más amplio de políticas que definen un sistema que debe seleccionar y diferenciar. La universidad sigue siendo un referente en la configuración de las políticas y de las prácticas escolares; el resto del sistema está bajo esta influencia constante y permanente, lo que hace necesaria la selección y construir para ello una gran variedad de categorías de éxito asociadas a ella que condiciona las prácticas de etapas anteriores (Fernández-Enguita, Mena-Martínez \& Riviere-Gómez, 2010).

El presente artículo aborda el análisis de las políticas públicas de apoyo educativo en España, Comunidad de Madrid, a partir del estudio desde una perspectiva etnográfica realizado durante siete años sobre un grupo de estudiantes a los que se les ha ofrecido una respuesta educativa, que ha combinado distintas alternativas legales disponibles: Programas de Educación Compensatoria, plan PROA (Programas de Refuerzo, Orientación y Apoyo) y Programas de Diversificación Curricular, tendente a revertir unas trayectorias escolares devaluadas, vulnerabilizadas, próximas al abandono escolar y, en consecuencia, a la exclusión educativa. De esta manera, se asume una mirada de las políticas desde lo que ellas suponen para los sujetos, que deben concretarlas mediante su actuación en sus respectivos contextos sociales y profesionales. Sin embargo, lejos de cualquier mirada tecnicista, esto supone una serie de procesos de apropiación y resignificación que resultan de máxima importancia para entender lo que termina sucediendo.

\section{Desafiliación-desligadura-exclusión y procesos de inclusión educativa}

En este artículo se busca poner el acento en los procesos que deshacen los vínculos de los sujetos con las instituciones y en aquellas prácticas que se ponen en funcionamiento con el fin de rehacerlos. De esta manera se entiende que lo que es muchas veces conceptualizado como fracaso o como abandono escolar es en realidad un complejo proceso de exclusión que "afecta profundamente a la estructura identitaria de los individuos" (Autès, 2004, p. 28). En este sentido, este autor llama desligadura al proceso por el cual una gran variedad de lazos construidos por los sujetos se desarman, se desmoronan. Así, si entendemos la escuela como una estructura portadora de sentido para la sociedad, la desligadura se produce cuando este sentido se pierde para los sujetos.

Por su parte, Robert Castel (2004) utiliza el término desafiliación para hacer referencia a procesos que generan desenganche y a determinados sujetos, la exclusión. Por tanto, es un concepto complementario al anterior, mientras pone la atención, en este caso, en aquello que produce la desligadura, la pérdida del sentido que las instituciones —en este caso la educativa - tienen para los sujetos. El autor identifica tres zonas de la vida social, que pueden ayudar a comprender los procesos de desafiliación en el ámbito educativo: a) una zona de integración, b) una zona de vulnerabilidad, de riesgo de exclusión, en la cual podemos identificar alertas que 
anticipan la desligadura y c) zona de exclusión, en la que los vínculos con la institución escolar se han roto y se ha producido la pérdida de su sentido.

Con estos conceptos, en este trabajo se pretende conectar con los rasgos de las políticas educativas, de los programas que de ellas se desprenden $y$, muy especialmente, de los procesos de apropiación y resignificación que intervienen tanto en los procesos de desafiliación-desligadura-exclusión, como en aquellos que generan procesos opuestos a estos. Los estudios que se han realizado hasta la fecha sobre las políticas de apoyo educativo o políticas compensatorias han ofrecido rasgos sobre su funcionamiento y resultados y pueden suponer aportes importantes para analizar y desarrollar este tipo de políticas o programas (Ferreira \& Teixeira, 2010; Picardo-João \& Victoria-Libreros, 2009). Por otra parte, ciertos aportes de la microetnografía han contribuido a la comprensión de las interacciones en el aula y su relación con los resultados escolares (Erickson, 2006; Koehler, 1978), lo mismo que los estudios de la continuidad y discontinuidad entre familia y escuela (Rodríguez-Izquierdo, 2011). Por su parte, los estudios macroetnográficos han contribuido a conectar los fenómenos escolares con el empleo, la economía y otras instituciones (Ogbu, 2006). Finalmente, autores como John U. Ogbu (2006) o Carlos Peláez-Paz (2013) sugieren un modelo ecológico-cultural, que integra ambas perspectivas.

En este caso se espera que el enfoque escogido, tanto teórico como metodológico, pueda ser un aporte en su intento de conectar las políticas educativas, sus concreciones en escenarios educativos singulares y sus efectos en los procesos de afiliación e inclusión educativa. Para ello resulta clave el concepto de trayectorias escolares, que hace referencia a la vida escolarizada de los sujetos, principalmente a la forma en que el sistema, la escolaridad, la cultura escolar y las prácticas educativas localizadas van configurando historias escolares concretas. En la trayectoria escolar, aunque la cultura familiar y la del grupo social, las condiciones de vida y la forma en que el sujeto mismo se enfrenta a la escuela son importantes, se asume una posición en la que, como sugiere Carlos Peláez-Paz (2013), el éxito o exclusión escolar no dependen de los rasgos o estados de las personas.

\section{Metodología}

La investigación se ha desarrollado desde una perspectiva etnográfica, la cual ofrece un marco epistemológico y metodológico apropiado y novedoso para el estudio de las políticas de apoyo educativo, coherente con nuestra concepción sobre las mismas. Esto resulta especialmente adecuado para abordar la forma en que los sujetos interactúan con las pres- cripciones normativas en un contexto específico: los centros escolares; conocer y producir interpretaciones sobre los procesos de traducción, apropiación, resignificación y desarrollo de acciones que tienen lugar en esta compleja trama cultural (Monarca, 2012).

Esta investigación se ha centrado en la respuesta educativa dirigida a un grupo de 11 estudiantes en una situación próxima al abandono escolar. La misma se ha desarrollado en un Instituto público de Enseñanza Secundaria (IES), ubicado en un pequeño pueblo de la sierra madrileña. Los informantes han sido los estudiantes antes mencionados y sus familias, el profesorado del centro y el profesorado específicamente implicado en la respuesta educativa objeto de estudio.

\section{Trabajo de campo}

El trabajo de campo se desarrolló durante cuatro años, aunque posteriormente se realizó el seguimiento de los estudiantes durante otros tres, hasta que lograron finalizar los estudios postobligatorios escogidos por cada uno de ellos. El investigador principal ocupó un rol participante durante los primeros cuatro años de la investigación, aunque esta participación se definió de forma diferenciada a lo largo de los mismos: a) durante tres años ocupó un rol profesional específico como orientador del instituto donde se desarrolló la investigación; b) durante el segundo año de la investigación ocupó además un lugar más específico, como tutor del grupo de estudiantes; c) el cuarto año asistió al instituto sin asumir ningún rol específico. Las posiciones a y b del investigador favorecieron enormemente el acceso al campo, a los contextos específicos de observación y a los informantes. Por otra parte, estas posiciones han ayudado a comprender muchas de las claves del funcionamiento de esta particular forma de "burocracia planificada" que, como sostiene Ángel Díaz de Rada-Brun (2008), caracteriza a este tipo de instituciones. Sin embargo, esto supuso un mayor cuidado del extrañamiento como condición imprescindible para la etnografía.

Aunque en etnografía no se suele hablar de secuencias o fases rígidas de la investigación, desde el punto de las intenciones de conocimiento de la misma es posible reconocer tres fases o momentos:

Fase 1: Centrada en el conocimiento de los procesos de configuración de las trayectorias escolares de los estudiantes implicados, antes de la puesta en práctica de la respuesta educativa específica que se ha comentado anteriormente.

Fase 2: Centrada en el desarrollo durante tres años de las acciones específicas tendentes a reencauzar las trayectorias escolares. Como se mencionó en la introducción, la respuesta educativa ha combinado de forma sistemática una serie de programas contempla- 
dos en la normativa vigente: A) El primer año, la respuesta educativa se organizó bajo la normativa que regula los Programas de Educación Compensatoria, concretamente en el denominado grupo específico de compensación educativa. Los objetivos principales de este primer año fueron dos: uno relacionado con el fortalecimiento de la autoestima y otro relacionado con el fortalecimiento académico. B) El segundo y tercer año de la respuesta educativa se enmarcaron en el Programa de Diversificación Curricular; el objetivo principal fue lograr el desarrollo de las competencias clave para la obtención del título de ESO. La organización globalizada de las áreas curriculares, por ámbitos, fue un rasgo característico tanto de este programa como del anterior. C) Finalmente, de forma transversal se ha implementado el Plan PROA, que ha garantizado el apoyo y seguimiento de los estudiantes fuera del horario escolar.

Fase 3: Enfocada en el análisis de las trayectorias escolares ya reencauzadas. Esta fase se ha desarrollado casi tres años después de que los 11 estudiantes terminaron la etapa de ESO, una vez finalizado el Ciclo Formativo de Grado Medio que cada uno de ellos había iniciado. El objetivo principal fue obtener una mirada de sus trayectorias escolares desde un momento significativamente distinto al de inicio de la investigación, marcada muy especialmente por la obtención de un título postobligatorio.

\section{Técnicas y procedimientos}

Se han empleado dos técnicas principales: la entrevista y la observación participante de tipo específico e inespecífico. Las entrevistas fueron realizadas en distintos momentos del proceso y se centraron, según los casos, en las intenciones de las fases antes mencionadas. Por su parte, tanto las observaciones específicas como las inespecíficas se realizaron a partir del rol participante del investigador, que fueron registradas en el diario de campo al finalizar los eventos de observación seleccionados como objeto de registro (desarrollo de clases, reuniones con tutores, juntas de evaluación, reuniones de seguimiento de los programas analizados, etc.). En estos casos, los registros de las observaciones se enfocaron en las acciones y las representaciones de los sujetos relacionadas con la apropiación y resignificación de la regulación normativa de los programas objeto de estudio.

De forma complementaria, se analizaron tres documentos clave: la programación del Grupo Específico de Educación Compensatoria, la Programación de Diversificación y el Plan PROA; se presupuso que constituyen un primer nivel de transformación de las prescripciones normativas. Junto con estos documentos, se analizaron también actas de las juntas de evaluación del grupo específico y del programa de diversificación. Finalmente, se realizaron tres grupos de discusión, todos ellos con los estudiantes objeto de la respuesta educativa objeto de estudio, uno en cada una de las fases antes explicadas.

Por último, en la fase tres de la investigación, se realizó una técnica muy sencilla con los estudiantes. La misma consistió en reflexionar y responder, con una calificación numérica, a cómo se habían sentido en los distintos momentos de su escolaridad. Para ello se les ofreció un esquema estructurado de acuerdo con las etapas y niveles educativos, desde $1^{\circ}$ Ciclo de Educación Primaria hasta el último año del Ciclo Formativo de Grado Medio (CFGM), en el cual tenían que reflejar numéricamente (escala 1-10) su valoración de cada uno de esos momentos. Se tomó la decisión de utilizar esta técnica, porque a lo largo de la investigación nos dimos cuenta de que a los estudiantes les resultaba más sencillo valorar sus sentimientos hacia su propia escolarización asignando puntuaciones que asignando palabras. Tanto las palabras como los números son producciones culturales y el impacto que la escuela había dejado en ellos en la incorporación de los números o calificaciones como forma de valorar los fenómenos, había sido muy alto. Por tanto, y teniendo en cuenta que ya contábamos con un gran número de relatos producto de las entrevistas y de las observaciones, se decidió avanzar con esta técnica.

\section{Procesamiento de la información}

Es preciso mencionar las transcripciones de las entrevistas realizadas y el diario de campo como bases principales de la información generada a lo largo de la investigación. Con respecto a las transcripciones, se realizó un proceso de análisis inductivo-deductivo, teniendo en cuenta que la misma entrevista ya estuvo enfocada hacia determinados ejes de indagación relacionados con los objetivos de la investigación.

En el diario de campo se reflejan operaciones de descripción, transformación e interpretación de los fenómenos; como sostienen Honorio Velasco y Ángel Díaz de Rada-Brun (1999, p. 130), el registro es ya "un proceso de selección y temporalización de la información, y por tanto, una elaboración reflexiva". A partir de allí, siguiendo a estos autores, se han ido extrayendo las categorías o contenidos analíticos que pasaron a formar parte de las tramas analíticas que aquí se ofrecen.

\section{Resultados}

Los resultados de la investigación serán expuestos a partir de lo recogido en las tres fases antes comentadas, cada una de las cuales ofrece una mirada 
desde un momento específico de la configuración de las trayectorias escolares. Esos momentos permiten establecer relaciones entre los rasgos de las mismas y los diversos actores, acciones, programas, políticas, que se vinculan a ellas; en cualquier caso y de acuerdo con lo ya explicado anteriormente, es una lectura no lineal, alejada de las interpretaciones causaefecto, una mirada que procura problematizar, interrogar e interpelar lo dicho y lo hecho, en el marco de las culturas de los centros y de las políticas que buscan otorgar una cierta direccionalidad a la realidad educativa.

En primer lugar se presentarán los resultados que se han obtenido a partir de la técnica antes explicada y aplicada a los estudiantes en la fase 3. A partir de esta técnica, los estudiantes otorgaron una puntuación de acuerdo a cómo se sintieron en cada una de las etapas educativas de su trayectoria escolar (escala 1-10) (gráfico 1).

Gráfico 1

Trayectoria emocional desde el punto de vista de los estudiantes

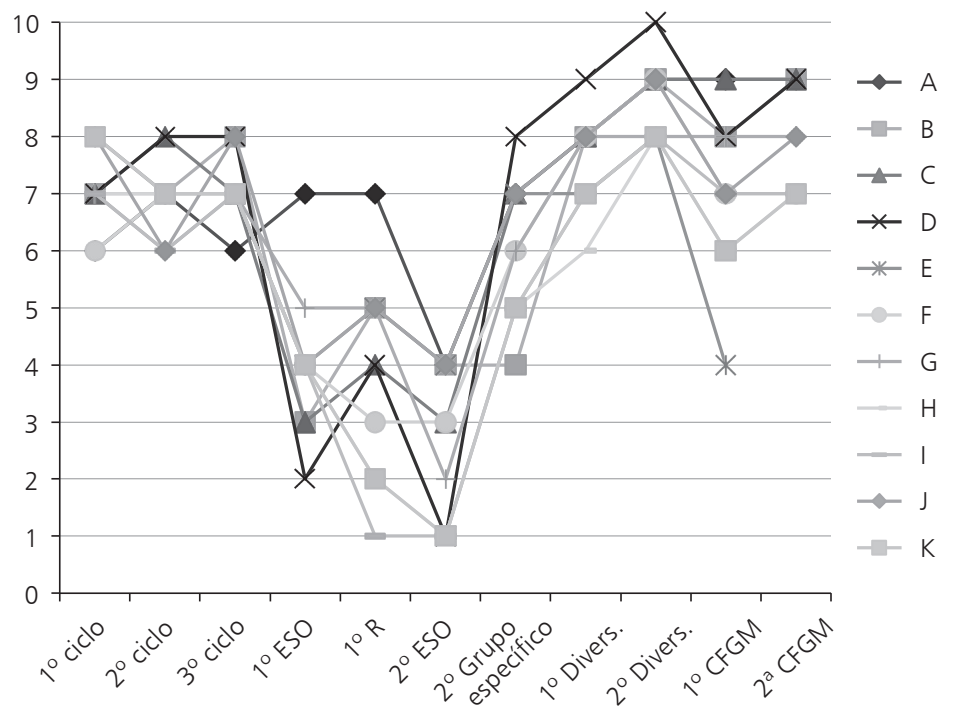

ESO: Educación Secundaria Obligatoria

Divers.: Programa de Diversificación

CFGM: Ciclos Formativos de Grado Medio

Fuente: elaboración propia

El gráfico ofrece una visión retrospectiva de los estudiantes y contribuye a visualizar sus trayectorias escolares desde su propia percepción, lo cual resulta clave para poder interpretar la información recogida en el trabajo de campo desde una lógica etnográfica. Los resultados de esta técnica, cuya intención ha sido objetivar los sentimientos de los estudiantes asociados a su escolarización, nos permite identificar tres momentos diferenciados: uno en Educación Primaria, otro entre $1^{\circ}$ y $2^{\circ}$ de ESO y un último momento que va desde el inicio del Programa de Diversificación hasta la finalización de los Ciclos Formativos de Grado Medio. Desde el punto de vista de la configuración de las trayectorias escolares se aprecian momentos de declive, en el cual los estudiantes se sienten emocionalmente alejados y apartados de lo escolar, algo que también podría relacionarse con el análisis de las calificaciones que han ido obteniendo; y otros 
de ascenso, en los que se sienten incluidos, forman parte de lo escolar y asumen que tanto la exclusión como la inclusión plena son categorías ideales que se presentan como extremos de procesos que, "en la práctica están interrelacionados" y se dan "entre ellos complejas combinaciones" (Peláez-Paz, 2013, p. 84). En este sentido, los resultados que se presentan aportan evidencias que contribuyen a explicar los procesos implicados en la configuración de estos momentos en relación con la respuesta educativa que se les ha brindado a los estudiantes.

\section{Fase 1: El proceso de desligadura}

De acuerdo con las evidencias obtenidas, se aprecia un cambio en las trayectorias escolares de los estudiantes al inicio de la etapa secundaria. Mientras es posible constatar un recorrido escolar normalizado en la Educación Primaria, que se refleja tanto en los relatos de los estudiantes como en sus propios expedientes académicos, el $1^{\circ}$ año de ESO se presenta para ellos y también para sus familias, como algo ambivalente. Por un lado, como oportunidad social y académica, aunque esto se da fundamentalmente durante un primer momento; por otro lado, como una brusca ruptura con la etapa anterior, como un problema, como una dificultad. Esta última representación ofrecida por los estudiantes es la que ha tomado más fuerza, en algunos de ellos a partir del tercer trimestre de $1^{\circ} \mathrm{ESO}$, en otros, a partir de $2^{\circ}$ ESO, según los casos. Las familias avalan en sus propios relatos estas imágenes y algo similar sucede con el profesorado.

Esta imagen que tienen los estudiantes sobre su inicio en la etapa secundaria se ve también reflejada en las actas de las juntas de evaluación y en sus propios expedientes académicos, cristalizados en el número de materias suspendidas y las repeticiones de curso. En cualquier caso, el proceso por el que se configuran estas representaciones es complejo y combina una gran variedad de aspectos de sus experiencias en el centro, en el aula: ideas y experiencias previas en educación primaria; ideas, experiencias y expectativas de su entorno familiar; su interacción con la nueva cultura de la ESO: concepciones, normas, valores, estilos de relación con los docentes y otros estudiantes, formas de enseñar, etc. En fin, todo lo relacionado con la estructura de tareas académicas y la estructura social de participación (Erickson, 2006).

De esta manera, antes de implementar la respuesta educativa enmarcada en la combinación de los programas mencionados, los datos evidencian una situación delicada desde el punto de vista académico y emocional, muy próximas al abandono escolar, relacionadas con la complejidad de factores recién mencionados. La información recogida permite afirmar que esta situación es producto de un proceso en el que han intervenido gran variedad de factores, los cuales, en este caso, se categorizan a partir de las respuestas ofrecidas por los informantes en la tabla 1.

Tabla 1

Factores explicativos del proceso de desenganche escolar

\begin{tabular}{|c|c|}
\hline Informante & Factores \\
\hline Estudiantes & $\begin{array}{l}\text { Dificultad para entender las explicaciones del profesor. } \\
\text { Abundancia de clase magistral, poca explicación alternativa. } \\
\text { Dificultad para seguir el ritmo de enseñanza. } \\
\text { Conocimientos muy alejados de su realidad. } \\
\text { Distancia con el profesorado. } \\
\text { Poca atención al que no entiende. } \\
\text { Poca preocupación por los estudiantes. } \\
\text { Excesiva cantidad de tareas y estudio para casa. } \\
\text { Poco apoyo fuera de la escuela para estudiar y hacer tareas. } \\
\text { Poco estudio. } \\
\text { Falta de habilidades de estudio. }\end{array}$ \\
\hline Familias & $\begin{array}{l}\text { Dificultades personales diversas por parte de sus hijos para responder a las exigencias académicas. } \\
\text { Distancia con el instituto. } \\
\text { Poco nivel previo para entender en el instituto. } \\
\text { Dificultades para apoyar a sus hijos con las tareas. }\end{array}$ \\
\hline Profesorado & $\begin{array}{l}\text { Poca motivación e interés de los estudiantes. } \\
\text { Poca atención en clase. } \\
\text { Mal comportamiento. } \\
\text { Conocimientos previos insuficientes. } \\
\text { Alta presión por cumplir los programas de estudio. }\end{array}$ \\
\hline
\end{tabular}

Fuente: elaboración propia 
Aunque no es posible realizar interpretaciones causales a partir de estos factores vistos de forma independiente por los propios informantes, sí se puede afirmar que todos ellos ofrecen indicios para interpretar el proceso de desenganche escolar de los estudiantes. De esta manera, se aprecian factores explicativos relacionados con lo didáctico-curricular, lo relacional, lo emocional y lo contextual. Más allá de la mirada singular que ofrece cada informante sobre ellos, estos factores pueden dar cuenta del carácter complejo y policausal de los procesos de desafiliación o desenganche de lo escolar.

Por otra parte, junto con lo recién presentado, se ha obtenido información específica sobre el papel que han podido tener los programas o acciones de apoyo educativo en los que habían participado los estudiantes en cuestión hasta ese momento. En este caso, todos habían recibido algún tipo de apoyo individualizado en pequeño grupo, ya sea por parte de la profesora de pedagogía terapéutica, de audición y lenguaje, de compensatoria o en alguna materia de refuerzo, y en algunos casos de forma combinada. En este sentido, es de destacar que, según se desprende de los relatos de los estudiantes, la mayoría de ellos no valoran positivamente este apoyo; solo destacan, en algún caso, la relación con la profesora responsable del mismo. Por otra parte, según lo comentado por los estudiantes y las mismas evidencias en cuanto a resultados escolares, estas respuestas muestran efectos más bien pobres. Por ejemplo, Silvia, una alumna del programa, afirmaba: "desde $1^{\circ}$ ESO me mandaron a apoyo, no sé para qué, me daban tonterías; todas cosas sueltas que no tenían nada qué ver con la clase. Lo peor de todo es que suspendía igual". De la misma manera, hay que destacar que, en muchos de los casos, estos programas de apoyo se sustentan en las teorías del déficit y la privación, según las cuales estamos ante problemas de los individuos (estudiantes, familias, etc.); los estudiantes en este caso, y desde esta perspectiva organizan una respuesta educativa individual, alejada de un enfoque ecológico-cultural (Ogbu, 2006) que amplíe la perspectiva interpretativa de las causas y la forma de responder a la situación.

En contraste con la opinión ofrecida por los estudiantes, es de destacar que los docentes sí valoran estos apoyos, porque ven en estas acciones una forma de ocuparse de estos alumnos; asumiendo, en algunos casos, que ellos no pueden, no saben o que no es de su competencia. La teoría del déficit antes mencionada subyace en muchas de sus explicaciones, por tanto, se asume que la respuesta debe ser individual. Junto con esta se ofrecen otras explicaciones, más relacionadas con la cultura académica de la ESO, históricamente asociada al tránsito hacia la universidad. Así, la ESO refleja una cultura escolar con rasgos que marcan importantes discontinuidades con la educación primaria: a) una enseñanza más expositiva y menos centrada en la actividad, b) más importancia por parte del profesorado a los resultados de un examen que a todo el trabajo desarrollado durante el trimestre, c) tareas para casa más centradas en el estudio que en el desarrollo de actividades o trabajos, d) un vínculo más distante entre profesores y estudiantes, e) una forma de regular el comportamiento distinta a la usada en la etapa anterior, f) escaso contacto entre profesorado y familias, etc.

Por su parte, en los relatos que ofrecen las familias, también subyace la teoría del déficit en relación con sus hijos y con ellas mismas pues reconocen que no pueden apoyarles. En este sentido, ofrecen una explicación similar a la que da el mismo centro escolar. Sin embargo, en sus relatos también aparecen otros elementos que pueden ser importantes para el análisis. La situación económica de las familias de los estudiantes 
era muy variada, desde aquellas que tenían negocios propios con buenos ingresos, hasta aquellas que estaban en precariedad socioeconómica. Pero tenían algo en común en cuanto a su capital cultural y formativo: ninguna había completado la enseñanza secundaria y algunos no habían terminado la enseñanza primaria. Por otra parte, todos otorgaban un valor simbólico a los estudios, a los títulos académicos, pero las familias con mejor situación económica no relacionaban su formación previa, su experiencia escolar, con su situación económica; mientras que aquellas que estaban en una situación más precaria sí consideraban que la falta de un título académico influía en esta situación. En ambos casos, para los padres y madres de estos estudiantes, la cultura académica de la ESO, aunque valorada, les era ajena, lejana; esto refleja una discontinuidad entre familias y escuelas de enseñanza secundaria.

\section{Fase 2: El proceso de reenganche}

Esta fase se ha centrado en la indagación de los procesos, acciones y representaciones relacionados con la respuesta educativa que se concreta en los programas enmarcados en las políticas de apoyo educativo. En este sentido, un primer aspecto a destacar, de acuerdo con la información obtenida, es que el proceso por el cual la propuesta ha cobrado forma se ha caracterizado por ser participativo, por implicar a profesores que voluntariamente quisieron asumir esta tarea, y por ser una programa que se había concebido a mediano plazo, con el objetivo de que todos los alumnos logren obtener el título de ESO y adquieran las competencias necesarias para seguir estudiando. Cabe destacar la confianza del profesorado participante en las posibilidades que esta respuesta educativa tenía para reencauzar las trayectorias escolares de los estudiantes. Sin embargo, ni esta confianza, ni las ideas con respecto al desarrollo de estos programas eran compartidos por todo el profesorado. De la misma manera, había disparidad de opiniones por parte de los otros informantes.

Por su parte, la variedad de opiniones del profesorado se ubica entre dos extremos: los que consideran que estos programas son necesarios y que deben existir y los que no; con una gran diversidad y, en ocasiones, disparidad de ideas sobre ellos. Lo mismo se aprecia en madres, padres y estudiantes. Algunos de ellos pensaban que estos programas eran para "alumnos que tenían problemas", no para sus hijos. Sin embargo, de la misma manera, creían que estos programas podían llegar a ser muy útiles si ayudaban a que se obtenga el título de la ESO. En cuanto a los estudiantes, antes de iniciar el programa, también coincidían en parte con estas apreciaciones; por un lado, circulaba la idea de que era para alumnos con problemas, pero lo valoraban en la medida en que pudiese contribuir a obtener el título de esta etapa educativa.

Más allá de las ideas en cuanto a la necesidad de los mismos, son clave en esta investigación los procesos que se han podido identificar de apropiación, resignificación y el desarrollo de acciones relacionadas con las políticas objetivadas en la normativa que regula estos programas. Sobre este aspecto, lo más valorado por parte de ciertos profesores es que la normativa contemple estos programas; que las políticas educativas den un margen de actuación al profesorado para implementar respuestas educativas contextualizadas. Uno de los orientadores del centro sostenía: "lo importante es que la ley habla de estos programas, que existen, y así tienes libertad para hacer lo mejor para los alumnos". Sin embargo, la normativa no es tenida en cuenta como reglamentación específica, no interesan tanto los detalles que ofrece, sino el marco global que genera y al que ellos suelen dar el contenido real. De hecho, la mayoría de los profesores que daban clase en el marco de esta respuesta educativa reconoce explícitamente que ni siquiera conoce la normativa que los regula.

En el análisis específico de la normativa referida a estos programas de apoyo educativo, se aprecia que no es tan precisa como en otros casos; por ejemplo, los Reales Decretos que regulan los currículos o los programas que de ellos se desprenden. En este sentido, la necesidad de su concreción es más evidente. Su localización se puede interpretar como el rasgo más relevante que los diferencia de los currículos basados en trayectorias teóricas. De acuerdo con las entrevistas y observaciones realizadas, precisamente esta necesidad de contextualización y concreción de la normativa ofrece la potencialidad para su éxito y abre las puertas para generar una propuesta ajustada a las trayectorias reales de los estudiantes. Este rasgo se relaciona con un factor mencionado por todos los profesores de estos programas, la adecuación del ritmo de enseñanza-ritmo de aprendizaje, es decir, realizar los ajustes necesarios entre los tiempos, los ritmos y los contenidos; algo imprescindible para ofrecer experiencias significativas de aprendizaje. Como expresaba Alejandro, uno de los profesores del programa: "aquí podemos ir a otro ritmo, podemos ir hacia atrás, empezar de muy abajo, y seguir desde ahí".

El profesorado destaca otro aspecto relacionado con el vínculo pedagógico, el interés por el otro, por el estudiante, el aspecto emocional, relacional. Marisa, una de las profesoras, daba máxima importancia a este aspecto: "antes de poder enseñar cualquier cosa, debes tener una buena relación con los alumnos, que se den cuenta [de] que les importas". Por su parte, también los estudiantes valoran notablemente este rasgo, "cuando empezamos con el grupo específico 
nos dimos cuenta [de] que los profesores querían que aprendamos, nos escuchaban, si necesitábamos, nos explicaban todas las veces necesarias" (Sergio).

En los procesos de implementación de estos programas también han aparecido como relevantes los siguientes aspectos: A) las expectativas de éxito de los profesores, es decir, la búsqueda de bienestar emocional y educativo, como se puede ver en las palabras de Rafael, un profesor del programa: "para trabajar bien ellos tienen que creer que pueden aprender y tienen que experimentarlo, tienen que aprender de verdad"; B) una tutoría fuerte: "ha sido clave el seguimiento personalizado, estar continuamente al lado de los alumnos y sus padres" (José, profesor del programa); C) las coordinaciones constantes e intensas: "el trabajo en equipo ha sido fundamental, siempre coordinándonos" (Beatriz, profesora del programa); D) la apropiación del programa por parte de los estudiantes; según las entrevistas, ellos lo han vivido como algo propio que les ha ofrecido contención, pertenencia, sentimiento de grupo, compañerismo y apoyo mutuo; E) el apoyo y seguimiento ofrecido fuera del horario escolar; aspecto especialmente valorado por los estudiantes y, sobre todo, por sus familias.

Finalmente, es posible resaltar que la configuración de la propuesta en su conjunto ha sido posible por una interpretación flexible y creativa de la norma, un convencimiento de que la escuela tiene una responsabilidad en la calidad de los resultados y que puede mejorarlos, y una fuerte implicación de orientadores, equipo directivo y un grupo concreto de profesores.

\section{Fase 3: Éxito escolar}

El nombre dado a esta fase no ofrece una valoración teleológica de la respuesta educativa en sí misma, que establezca una relación medios-fines. Tampoco refleja un momento concreto, la fase anterior ha procurado ofrecer mayor información sobre los procesos que han permitido reencauzar las trayectorias de los estudiantes. En este caso, se trata del reflejo de un estado de bienestar expresado por los estudiantes, opuesto al expresado al inicio de la investigación por ellos mismos; es una imagen obtenida casi tres años después de haberles ofrecido la respuesta educativa específica que ha intentado reencauzar sus trayectorias escolares. Esta imagen, junto con la realidad que representa, constituye una forma de valorar y evaluar la respuesta educativa analizada en la fase 2 con un referente externo, un título postobligatorio. En este sentido, todos los relatos de los estudiantes coinciden en señalar que la respuesta educativa que aquí se ha analizado, ha sido una experiencia de éxito. Esta imagen de éxito, de acuerdo con sus propios relatos, se sostiene en tres elementos clave:

1. En el simbolismo que tiene para ellos y su familia haber conseguido un título postobligatorio. Ramón, uno de los estudiantes del programa, dijo en la entrevista: "mi familia nunca estudió, mi hermano lo dejó; soy el primero que tengo un título importante". En todos los casos, estamos ante una primera generación de jóvenes que, desde el punto de vista intrafamiliar, obtienen un título, no solo de enseñanza secundaria, sino también de enseñanza postobligatoria.

2. El simbolismo social que tiene haber conseguido esta titulación. Cada vez se insiste más en este aspecto como un elemento clave de la calidad de los sistemas educativos. Un padre expresó en la entrevista, "hoy sin estudio no eres nadie, aunque tengas dinero; 
si no tienes cultura, estás muy perdido, no lo aprovechas igual". Algo similar dijo María, otra de las madres, "continuamente escuchamos en la TV lo importante que es estudiar".

3. Finalmente, el éxito es una representación vinculada a haber experimentado la superación de una situación que, en un momento de sus trayectorias vitales, daban por perdida. Sara lo explicaba de esta manera, "yo nunca pensé que iba a terminar un Ciclo Formativo, ni siquiera la ESO; si no hubiese hecho diversificación, hubiese abandonado".

\section{Discusión}

En este artículo se ha analizado la configuración de unas determinadas trayectorias escolares a partir de lo dicho y lo hecho por una serie de actores sociales: profesores, padres, madres y estudiantes de un IES. La investigación se inicia en un momento particular de estas trayectorias, muy próximas al abandono escolar, hecho que muy posiblemente haya condicionado las interpretaciones de los informantes acerca de la respuesta educativa que se implementó, como concreción de las políticas de apoyo educativo, para enfrentar la situación de desenganche, explicada en la fase 1. Estas rupturas con los lazos que dan sentido a lo social, tal como sostienen Michel Autès (2004) y Robert Castel (2004), forman parte de los procesos que explican la exclusión de los sujetos. Como reflejan los textos de los autores recién mencionados, estos procesos de desafiliación, desenganche o exclusión tienen lugar en el marco de un sistema del cual estos procesos forman parte. Por otro lado, desde el punto de vista de las políticas, de la supuesta direccionalidad que estas pretenden dar a la realidad, los procesos de desafiliación siempre hacen referencia, de alguna manera, a una peculiar interacción entre estas prescripciones normativas y los mecanismos de apropiación, resignificación y acción de los actores.

De esta manera, las trayectorias escolares de los estudiantes de esta investigación, como producto de esta compleja interacción, junto con las representaciones que los diversos actores tienen acerca de las mismas, se transforman en un potente analizador de estas políticas. Sin embargo, lejos de cualquier interpretación simplista y lineal, la trama producida en esta investigación ofrece una mirada compleja y en ocasiones contradictoria. En este sentido, podemos apreciar que, incluso en unos mismos actores, las representaciones que circulan en torno a cómo se han configurado las trayectorias de estos estudiantes, por qué ha pasado, qué se debe hacer, etc., evidencian la existencia de concepciones, creencias, prácticas, etc., que muestran un mundo escolar en continua tensión. Esto queda reflejado, por ejemplo, en los factores explicativos del desenganche escolar que ofrece cada uno de los informantes.

Por otra parte, esta tensión expresada en las diversas normas que regulan la institución escolar, también se refleja en el contexto social y escolar estudiado. Los padres, el profesorado y los estudiantes lo muestran en sus discursos y sus acciones. Como ha quedado en evidencia, esto se puede apreciar en las explicaciones que unos y otros ofrecen sobre estas trayectorias desenganchadas y sobre los programas específicos para actuar sobre ellas. Así, algunas familias ven con cierto recelo estos programas, mientras que simultáneamente los valoran y solicitan como una alternativa para alcanzar sus expectativas: "que sus hijos titulen", algo que, por otro lado, refleja el valor que dan a la educación secundaria. Por su parte, hay profesores que los valoran y otros que los rechazan, con diversos matices entre ambos extremos, y en ocasiones hay quienes asumen ambas posiciones. 
Quizá sean precisamente estos rasgos — la tensión y la contradicción- los que mejor definen la existencia de estos programas, derivados de las políticas de apoyo educativo, pero también, por medio de ellas, de todo el sistema escolar. Su presencia nos indica que las políticas pensadas para un sujeto abstracto, que prescriben trayectorias teóricas ideales (Terigi, 2010), por paradójico que pudiera parecer, van generando o reforzando diferencias ya existentes en la estructura social y en las propias trayectorias de los estudiantes. El sistema escolar, los centros educativos y las prácticas docentes están continuamente atravesados, tensionados, por lo que Flavia Terigi (2010) denomina monocronía, rasgo que hace referencia a que la enseñanza y el aprendizaje están estrictamente sujetos a un mismo programa, que se desarrolla con arreglo a unos tiempos predeterminados, a un mismo ritmo, en unas mismas secuencias, en unos mismos grupos, en una edad determinada, bajo una misma secuencia de contenidos, unas mismas tareas, una misma evaluación, en un mismo momento (Edelstein, 2010; Tenti, 2011). Tal como ha sido puesto en evidencia a partir de los datos obtenidos, la fase 1 muestra que estos rasgos del funcionamiento escolar están dentro de los factores explicativos del desenganche de los estudiantes. Sin embargo, las mismas políticas contemplan alternativas, pero refuerzan la idea de que dar una respuesta ajustada al contexto y a los sujetos de aprendizaje debe ser una excepción. Desde este punto de vista, estos programas no solo interpelan la monocronía recién explicada, sino que suponen su clara alteración. Suponen un desafío a la cultura académica de la ESO que, en su funcionamiento actual, refuerza la discontinuidad con la educación primaria y con aquellas familias con menos años de escolarización, lo cual genera una ruptura en la concreción de la misma idea de educación básica que se ha querido plasmar en el marco de los procesos democratizadores de la educación.

De esta manera, estos programas agregan tensión y contradicción, ante un sistema general que produce, de acuerdo con esta investigación, desenganche, desafiliación y, de acuerdo con los aportes de otras investigaciones, también abandono y exclusión (Escudero-Muñoz, 2009). Estos programas ofrecen, explicado de forma simple, cuando menos, presencia $y$, en ocasiones, como en el caso investigado, aprendizaje y participación (Echeita-Sarrionandia, 2008). Para ello, de acuerdo con los resultados obtenidos, el papel del profesorado en la apropiación de las políticas de apoyo educativo es clave para que la respuesta educativa ofrecida a los estudiantes tenga los efectos buscados.

En este sentido, es llamativa la forma en que los profesores se han apropiado de las prescripciones normativas. Mientras que las prescripciones generales se asumen con una lógica de aplicación lineal que acompaña y sostiene la monocronía antes mencionada, en estos programas, la norma apenas es tenida en cuenta. Las mismas características de la normativa vinculada con ellos lleva a la necesidad de asumir mayor protagonismo a la hora de definir la propuesta concreta; para ello, se ponen en juego otro tipo de prácticas: reuniones, debates, seguimientos y evaluaciones, etc., en los cuales el protagonismo docente y sus conocimientos específicos resultan imprescindibles.

Se puede apreciar así que la inclusión y la exclusión se dan dentro del mismo sistema como dos rasgos ideales (Peláez-Paz, 2013), las trayectorias escolares se configuran por medio de complejos procesos entre ambos polos. Estos fenómenos pueden ser explicados de forma simultánea, tal como se ha podido ver, por rasgos estructurales del sistema, la cultura social, familiar y escolar en particular, las prácticas que se desarrollan dentro 
de los centros educativos y sus aulas y por la forma en que los actores se apropian de los procesos y generan acciones y prácticas a partir de estas apropiaciones y resignificaciones.

\section{Conclusiones}

Como se ha podido ver, la existencia y necesidad de estas políticas de apoyo educativo ponen en evidencia una continua tensión en el funcionamiento escolar entre lo normalizado, lo homogéneo y lo diverso. Todas estas políticas se dirigen a estudiantes específicos y hacen una definición cerrada del perfil de sus supuestos beneficiarios, mientras que las políticas ordinarias, para denominar aquellas dirigidas a todos, definen un inexistente perfil abstracto de un estudiante descontextualizado. De esta manera, el lugar para la diversidad, para la diferencia, aparece con frecuencia, desde el punto de vista normativo, asociado a una disfuncionalidad o a un déficit que suele ser ubicado en los sujetos o en las instituciones. Este hecho supone un obstáculo para una mirada inclusiva de las prácticas educativas, en primer lugar porque hacen pensar lo diverso como excepcional; en segundo lugar porque inducen respuestas educativas en itinerarios diferenciados. Así, desde el punto de vista normativo se refuerza una idea teórica de las trayectorias escolares, que predomina también en las representaciones de profesorado, estudiantes y familias.

Sin embargo, como se desprende del análisis de los datos, estos programas funcionan como un paraguas bajo el cual es posible realizar todo aquello que sea necesario para que los alumnos aprendan, incluso, como se ha explicado, alterar tiempos, ritmos, secuencias, metodologías, organización del aula; en síntesis, el orden que la enseñanza fuera de estos programas tiene para la enseñanza. De esta manera, las respuestas ajustadas al sujeto y al contexto de aprendizaje quedan reservadas para situaciones excepcionales; mientras no se confirme este hecho, se mantiene la respuesta homogénea, con saberes y experiencias deslocalizadas, prácticas que, como se ha podido ver, tienen efectos claros en la configuración de las trayectorias escolares de determinados estudiantes (Monarca, Rappoport \& Fernández-González, 2012). El porqué en estos programas se acepta la alteración de la monocronía y no fuera de ellos, es una pregunta que merece ser profundizada en nuevas investigaciones.

El último aspecto que se quiere destacar en este caso, rescatando la voz de los estudiantes, es el valor que este tipo de programas puede tener para las trayectorias escolares y vitales de los sujetos. Más allá de cualquier análisis del sistema escolar, la vida cotidiana de las personas está atravesada por múltiples entornos y prácticas, las cuales ejercen diversas influencias. De acuerdo con los relatos de los estudiantes, el mismo sistema les ha ofrecido "lo mejor" y "lo peor", así de polarizada representan ellos su experiencia escolar. Dentro de esta representación, la respuesta educativa implementada es la que los reengancha a lo escolar y, desde una mirada más a largo plazo, con su título de enseñanza postobligatoria conseguido, la que les ha permitido llegar a ese lugar impensable para ellos cuando iniciamos la fase 1 de la investigación.

\section{Sobre el autor}

Héctor Monarca es profesor-investigador en la Universidad Autónoma de Madrid, UAM, departamento de Didáctica y Teoría de la Educación. Miembro del grupo de investigación sobre Políticas Educativas Supranacionales (GIPES). Doctor en educación, Universidad Complutense de Madrid. Licenciado en ciencias de la educación, Universidad de Buenos Aires. Profesor para la enseñanza primaria. 


\section{Referencias}

Autès, Michel (2004). Tres formas de desligadura. En Saül Karsz (coord.). La exclusión: bordeando sus fronteras. Definiciones y matices, 15-59. Barcelona: Gedisa.

Castel, Robert (2004). Encuadre de la exclusión. En Saül Karsz (coord.). La exclusión: bordeando sus fronteras. Definiciones y matices, 55-86. Barcelona: Gedisa.

Díaz de Rada-Brun, Ángel (2008). ¿Qué obstáculos encuentra la etnografía cuando se practica en las instituciones escolares? En María Isabel JocilesRubio \& Adela Franzé-Mudano (eds.). ¿Es la escuela el problema? Perspectivas socio-antropológicas de etnografía y educación, 24-48. Madrid: Trotta.

Diker, Gabriela (2010). Entre la ciencia ficción y la política. Variaciones sobre la desaparición de lo escolar. En Graciela Frigerio \& Gabriela Diker (comps.). Educar: saberes alterados, 149-163. Paraná, Argentina: Fundación La Hendija.

Dubet, François (2005). Exclusión social, exclusión escolar. En Julián J. Luengo (comp.). Paradigmas de gobernación y de exclusión social en la educación. Fundamentos para el análisis de la discriminación escolar contemporánea, 93-115. Barcelona: Ediciones Pomares.

Dubet, François \& Martuccelli, Danilo (2000). ¿En qué sociedad vivimos? Buenos Aires: Losada.

Echeita-Sarrionandia, Gerardo (2008). Inclusión y exclusión educativa. "Voz y Quebranto". REICE, Revista Electrónica Iberoamericana sobre Calidad, Eficacia y Cambio en Educación, 6 (2), 9-18. Disponible en: http://www.redalyc.org/ pdf/551/55160202.pdf

Edelstein, Gloria (2010). Enseñanza, políticas de escolarización y construcción didáctica. En Graciela Frigerio \& Gabriela Diker (comps.). Educar: ese acto político, 139-152. Paraná, Argentina: La Hendija. Disponible en: http://www.amsafe. org.ar/formacion/images/2013-CursoDirectores/ Eje5/Gloria\%20Edelstein\%20-\%20Enseanza\%20 polticas $\% 20$ de $\% 20$ escolarizacin $\% 20 y \% 20$ construccin\%20didctica.pdf

Erickson, Frederick (2006). El discurso en el aula como improvisación: las relaciones entre la estructura de la tarea académica y la estructura de la participación social en clase. En Honorio Manuel Velasco-Maíllo, Francisco Javier García-Castaño \& Ángel Díaz de Rada-Brun (eds.). Lecturas de antropología para educadores. El ámbito de la antropología de la educación y de la etnografía escolar, 325-353. Madrid: Trotta.
Escudero-Muñoz, Juan Manuel (2009). Buenas prácticas y programas extraordinarios de atención al alumnado en riesgo de exclusión educativa. Profesorado. Revista de Currículo y Formación del Profesorado, 13 (3), 107-141. Disponible en: http://www.ugr.es/ recfpro/rev133ART4.pdf

Fernández-Enguita, Mariano; Mena-Martínez, Luis \& Riviere-Gómez, Jaime (2010). Fracaso y abandono escolar en España. Barcelona: Obra Social Fundación La Caixa, Colección Estudios Sociales 29. Disponible en: http://smartgroups. es/iesestuaria/wp-content/uploads/2015/06/ Fracaso-Escola-_ObraSocialLaCaixa.pdf

Ferreira, Isabel \& Teixeira, Ana Rita (2010). Territórios educativos de intervençao prioritária: breve balanço e novas questões. Sociologia: Revista da Sociologia da Faculdade de Letras da Universidade do Porto, FLUP, 20, 331-350. Disponible en: http://ler.letras.up.pt/uploads/ficheiros/8804. pdf

Koehler, Virginia (1978). Classroom Process Research: Present and Future. Journal of Classroom Interaction, 13 (2), 3-11.

Ministerio de Educación, Cultura y Deporte, MECD (2015). Sistema estatal de indicadores de la educación 2015. Madrid: Secretaría General Técnica, Subdirección General de Documentación y Publicaciones. Disponible en: http://www.mecd. gob.es/mecd/dms/mecd/servicios-al-ciudadanomecd/estadisticas/educacion/indicadores-publi caciones-sintesis/sistema-estatal-indicadores/ SEIE_2015.pdf

Monarca, Héctor (2011). La escuela fragmentada. Revista Iberoamericana de Educación, 57, 203-215. Disponible en: http://rieoei.org/rie_contenedor. php? numero $=$ rie $57 \&$ titulo $=$ RIE\%2057\%20Sep tiembre-Diciembre\%20/\%20Setembro-Dezem bro\%202011

Monarca, Héctor (2012). La investigación educativa desde un enfoque etnográfico. Fundamentos epistemológicos. Sevilla: Fénix. Disponible en: https://repositorio.uam.es/bitstream/hand le/10486/666954/investigacion_monarca_2012. pdf? sequence $=1$ \&isAllowed $=y$

Monarca, Héctor; Rappoport, Soledad \& FernándezGonzález, Antonio (2012). Factores condicionantes de las trayectorias escolares en la transición entre enseñanza primaria y secundaria. Revista Española de Orientación y Psicopedagogía, REOP, 23 (3), 49-62. Disponible en: http://www2.uned. es/reop/pdfs/2012/23-3\%20-\%20Monarca.pdf

Ogbu, John U. (2006). Etnografía escolar. Una aproximación a nivel múltiple. En Ángel Díaz de Rada-Brun, Honorio Manuel Velasco-Maíllo, Francisco Javier García-Castaño (eds.). Lecturas de antropología 
para educadores. El ámbito de la antropología de la educación y de la etnografía escolar, 145-174. Madrid: Trotta.

Peláez-Paz, Carlos (2013). La infancia pobre, marginada y excluida. Aportaciones de la etnografía a la praxis socioeducativa. Educación y Futuro, 29, 81-105. Disponible en: https://dialnet.unirioja.es/servlet/ articulo?codigo $=4456838$

Pericacho-Gómez, Francisco Javier (2014). Pasado y presente de la renovación pedagógica en España (de finales del siglo XIX a nuestros días). Un recorrido a través de escuelas emblemáticas. Revista Complutense de Educación, 25 (1), 47-67. Disponible en: http://revistas.ucm.es/ index.php/RCED/article/view/43309/41007

Picardo-João, Óscar \& Victoria-Libreros, Julián A. (2009). Educación acelerada. El Salvador. Madrid: OEI.

Rodríguez-Izquierdo, Rosa María (2011). Discontinuidad cultural. Estudiantes inmigrantes y éxito académico. Aula Abierta, 39 (1), 69-80. Disponible en: https://dialnet.unirioja.es/servlet/articulo?codigo=3353887

Tenti-Fanfani, Emilio (2011). La escuela y la cuestión social. Ensayos de sociología de la educación. Buenos Aires: Siglo XXI.

Terigi, Flavia (2010). El saber pedagógico frente a la crisis de la monocronía. En Graciela Frigerio \& Gabriela Diker (comps.). Educar: saberes alterados, 99-110. Paraná, Argentina: Fundación La Hendija. Disponible en: http://www.unter.org.ar/imagenes/TERIGI-\%20EL\%20SABER\%20PE DAGOGICO001_0.pdf

Velasco, Honorio \& Díaz de Rada-Brun, Ángel (1999). La lógica de la investigación etnográfica. Un modelo de trabajo para etnógrafos de escuela. Madrid: Trotta.

Vitar, Ana (2006). Las políticas y lo(s) político(s). En Ana Vitar (coord.). Políticas de educación. Razones de una pasión, 25-49. Buenos Aires: Miño y Dávila. 